\title{
Head-to-head comparison of protocol modifications for the generation of collagen-induced arthritis in a specific-pathogen free facility using DBA/1 mice
}

\author{
Sherry Thornton ${ }^{1}$ and Richard T. Strait ${ }^{2}$ \\ Department of Pediatrics and Cincinnati Children's Hospital Medical Center, Divisions of ${ }^{1}$ Rheumatology \\ and ${ }^{2}$ Emergency Medicine, University of Cincinnati College of Medicine, Cincinnati, $\mathrm{OH}$
}

BioTechniques 60:119-128 (March 2016) doi 10.2144/000114388

Keywords: collagen-induced arthritis (CIA); lipopolysaccharide (LPS); complete Freund's adjuvant (CFA); incomplete Freund's adjuvant (IFA); specific-pathogen free (SPF)

Collagen-induced arthritis (CIA) is a widely used mouse model for studying inflammatory arthritis (IA). However, CIA induction protocols differ between laboratories, and direct comparison between protocol variations has not been reported. To address this issue, DBA/1 mice housed in conventional and specific-pathogen free (SPF) facilities were administered various combinations of two doses of collagen type II (CII) in complete (CFA) or incomplete Freund's adjuvant (IFA); some mice were also injected with lipopolysaccharide (LPS) and/or additional Cll at specific intervals. Mice were evaluated for IA over the subsequent 2 months. Depending directly on the combination of $\mathrm{Cll}$, CFA, IFA, and LPS used, the incidence of IA ranged between 20\%-100\%, and severity extended from mild to severe even in an SPF environment. Our results demonstrate for the first time in head-to-head comparisons that specific variations in the use of CII, CFA, IFA, and LPS can induce a range of arthritic disease intensity and severity in an SPF facility. Thus, distinct experimental settings can be designed for robust assessment of factors that either exacerbate or inhibit arthritis pathogenesis. Furthermore, by achieving 100\% incidence in an SPF facility, the protocols provide a practical and humane benefit by reducing the number of mice necessary for experimental assessment.

Collagen-induced arthritis (CIA) in mice provides an experimental model for analysis of the pathophysiology and histopathology of human inflammatory joint diseases including rheumatoid arthritis (RA). CIA shares many pathological, immunological and genetic susceptibility features with RA (1-7). Indeed, current frontline therapeutics that are now part of the standard of care for RA (e.g., tumor necrosis factor (TNF) and TNF-receptor inhibitors) were characterized and validated using the CIA model in pre-clinical studies (8-12). Additionally, the CIA model has aided in verifying the significance of newly appreciated human RA findings and also guides our understanding of future therapeutic interventions (13-15).

CIA protocols vary from laboratory to laboratory, including composition and timing for delivery of the antigen (Ag) and adjuvant, and the use of lipopolysaccharide (LPS). The most commonly used $\mathrm{Ag}$ is bovine collagen type II (CII), although Cll from other animals can also induce arthritis $(2,6,16)$. The use of complete (CFA) or incomplete (IFA) Freund's adjuvant is discrepant among protocols, with either one or two injections being necessary to show significant disease (10-12,17-20). Hygiene of the colony appears to be an especially important factor contributing to the development of inflammatory arthritis (IA). Specific-pathogen free (SPF) facilities appear to be less conducive than conventional facilities to induction of $\mathrm{ClA}$, as experienced by us and reported by others (20). In similar settings of poor induction of CIA, some investigators have used LPS to further potentiate the effects of Cll immunization; however, use and timing of LPS treatment is quite variable $(7,16,21-25)$.

To date, no publications offer a direct comparison of the variations in IA incidence and severity between conventional and SPF facilities or different arthritis induction protocols involving CFA, IFA, LPS, and multiple $C l l$ doses. Here we examine whether exposure to LPS enhances development of IA and whether additional Cll injections with or without LPS after initial immunizations with Cll in CFA (CII/CFA) elicit a more consistent arthritic response in an SPF setting. Furthermore, substitution of IFA for CFA is directly compared within these protocols, as this is an important issue for laboratory animal welfare. With specific changes in the protocol, incidence and severity of IA ranges from severe arthritis in $100 \%$ of mice to minor

\section{METHOD SUMMARY}

The severity and incidence of collagen-induced arthritis (CIA) in DBA/1 mice can be manipulated by variations in collagen type II (CII), complete Freund's adjuvant (CFA), incomplete Freund's adjuvant (IFA), and lipopolysaccharide (LPS) usage to result in a desired effect; from severe arthritis in $100 \%$ of mice treated to mild arthritis in less than $20 \%$ of the mice. These variations are applicable to a specific-pathogen free (SPF) facility and allow investigators to better design experiments to maximize utility of results while limiting the number of animals used. 


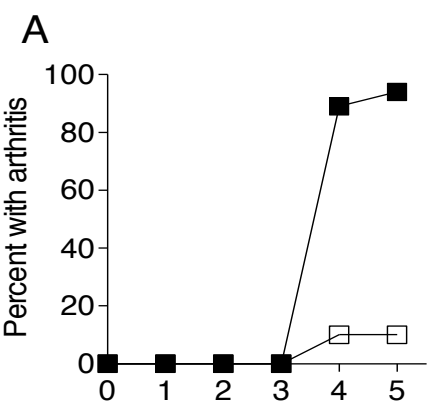

Weeks after first priming dose
$\mathrm{B}$

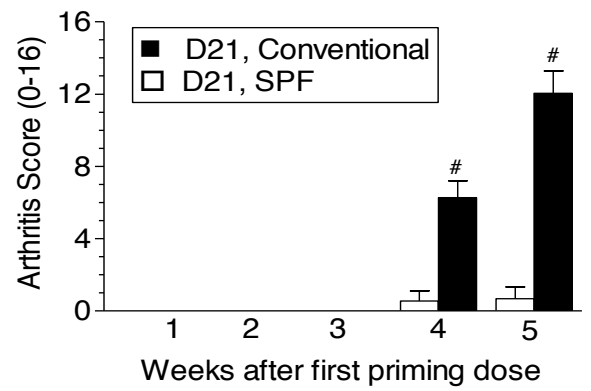

C

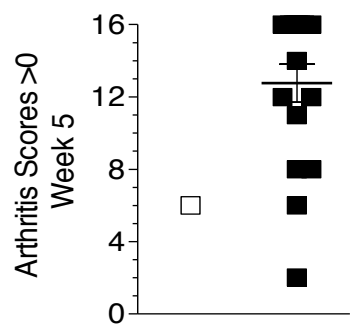

Figure 1. The D21 protocol does not invoke any significant arthritis in DBA/1 mice housed in a specific-pathogen free (SPF) colony compared with a conventional colony. DBA/1 mice, housed either in a conventional colony $(n=18)$ or an SPF colony $(n=10)$, were immunized as per the protocol D21 and followed for the development of arthritis. Arthritis was defined as the development of joint swelling and was measured as (A) the percentage of mice that have any swollen joints. The average clinical arthritis score (mean $\pm \mathrm{SEM}$ ) for each group is shown in (B). \# $P$-value $<0.005$. (C) Final arthritis scores in week 5 for only those mice that developed arthritis.

arthritis occurring in $>20 \%$ of the mice. This ability to manipulate the incidence and severity of IA allows investigators to better evaluate genetic contributions and treatment factors that can either enhance or diminish disease. Furthermore, our observations suggest that IFA can be substituted for CFA when given in combination with additional doses of LPS and CII with significant disease incidence and severity still observed, thus alleviating concerns regarding CFA injections in this animal model.

\section{Materials and methods}

Animals

Six-week old DBA/1 male and female mice were purchased from The Jackson Laboratory (Bar Harbor, ME) and housed in either a conventional colony or an SPF colony with a 12-h light/dark cycle and access to food and water ad libitum. All animal studies were approved by the Institutional Animal Care and Use Committee in accordance with guidelines of the National Institutes of Health.

Arthritis induction

Bovine Cll was purchased from Elastin Products Co, Inc. (Owensville, MO) and stored at $-80^{\circ} \mathrm{C}$ as a $2 \mathrm{mg} / \mathrm{mL}$ solution in $0.1 \mathrm{M}$ acetic acid in small aliquots, avoiding freeze-thaw cycles. IFA and Mycobacterium tuberculosis were purchased from Difco (Lawrence, KS) through Fisher Scientific (Waltham, MA). CFA was made under a sterile hood by mixing $M$. tuberculosis, crushed by a sterile mortar and pestle, with IFA to a final concentration of 2 mg M. tuberculosis/1 mL IFA and vortexing the mixture vigorously for 3 min before storing it at $4^{\circ} \mathrm{C}$. On the day of the injection, specific volumes of $\mathrm{Cll}$ and CFA or IFA were drawn up into separate $5 \mathrm{~mL}$ glass syringes through 18 gauge needles so that the contents of the 2 syringes when mixed together yielded a final Cll concentration of $1 \mathrm{mg} / \mathrm{mL}$. Both syringes were attached to either end of a micro-emulsifying needle and mixed together by passing their contents back and forth through the emulsifying needle 100 times. Emulsified solution was placed on ice until used and pushed through the emulsifying needle again several times just prior to use. One hundred microliters emulsified solution was injected intradermally (i.d.) near the base of the tail of an anesthetized mouse. On the day of the injection, the Cll and LPS (lyophilized powder from Salmonella enterica; Sigma, St. Louis, $\mathrm{MO}$ ) solution was mixed by vortex to a final ratio of $100 \mu \mathrm{g} \mathrm{Cll}$ and $50 \mu \mathrm{g}$ LPS in a final volume of $200 \mu \mathrm{L}$. This solution was injected i.d. near the hindquarters avoiding any area with visible scarring from a previous injection site.

Macroscopic assessment of arthritis incidence and severity

Mice were scored for presence of macroscopic arthritis the day of the initial injection and then 3 times a week starting on day 14. Severity of arthritis was scored using an arthritic index scoring system ranging from 0 to 4 per paw $(0=$ no detectable arthritis, $1=$ swelling and/or redness of paw or 1 digit, 2 = two digits involved, $3=$ three digits involved, and 4 = severe arthritis of the entire paw and all digits) with a maximum score of 16 per mouse (26).

\section{Histological analysis of arthritis}

To evaluate for arthritis beyond the paws, mice were sacrificed, typically on day 56 post injection, and posterior knees were harvested. Knees were immediately placed in formalin for a minimum of $72 \mathrm{~h}$, decalcified in EDTA, paraffin embedded, and processed in the usual manner for hematoxylin and eosin (H\&E) staining (27). Histological slides from the center of the knee joint were scored in a blinded fashion using a histopathologic scoring system as previously published (27). In brief, the scoring system has 5 categories: inflammation (score 0-3), synovial hyperplasia (score 0-3), pannus (score 0-1), edema (score 0-3), and bone/cartilage loss (score 0-3). Scores were summed to determine the total histopathology index per knee per mouse (score 0-13). Higher scores represent more severe pathology.

\section{ELISA analysis of Cll-specific titers} Costar 96-well flat bottom ELISA plates (Bio-Rad, Hercules, CA) were coated with

Table 1. Summary of protocols: Timing for injection of reagents.

\begin{tabular}{|c|c|c|c|c|c|c|c|}
\hline \multirow{2}{*}{ Protocol name } & \multicolumn{7}{|c|}{ Treatment on specific day } \\
& Day 0 & Day 14 & Day 17 & Day 21 & Day 24 & Day 28 & Day 42 \\
\hline D21 & CII/CFA & - & - & CII/CFA & - & - & - \\
\hline D21+LPS & CII/CFA & & & CII/CFA & LPS & & \\
\hline D14 & CII/CFA & CII/CFA & LPS & - & - & - & - \\
\hline D14+LPS/CII & CII/CFA & CII/CFA & LPS & - & - & LPS/CII & LPS/CII \\
\hline CFA/IFA+LPS/CII & CII/CFA & CII/IFA & LPS & - & - & LPS/CII & LPS/CII \\
\hline IFA/IFA+LPS/CII & CII/IFA & CII/IFA & LPS & - & - & LPS/CII & LPS/CII \\
\hline IFA/IFA+CII & CII/IFA & CII/IFA & LPS & - & - & CII & CII \\
\hline IFA/IFA+LPS & CII/IFA & CII/IFA & LPS & - & - & LPS & LPS \\
\hline collagen type II (CII); Complete Freund's adjuvant (CFA); incomplete Freund's adjuvant(IFA); lipopolysaccharide (LPS) \\
\hline
\end{tabular}


A

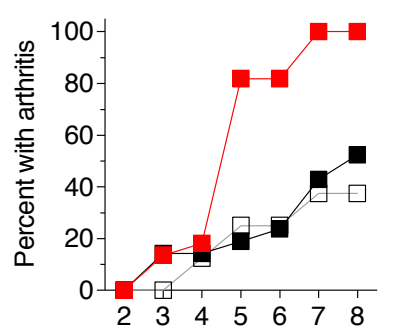

Weeks after first priming dose
B

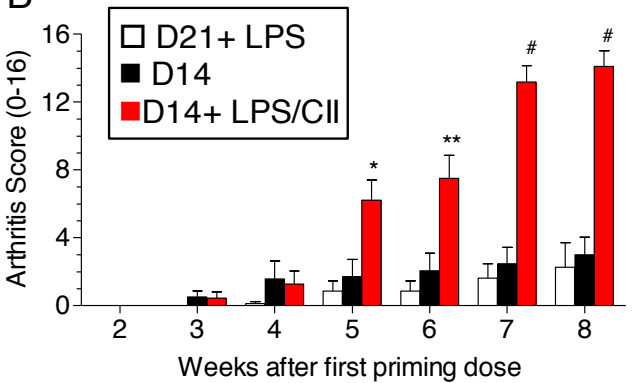

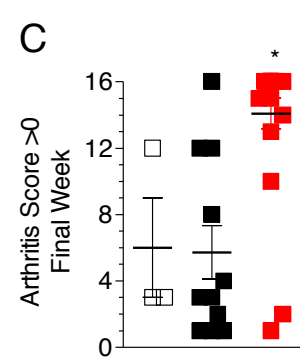

D

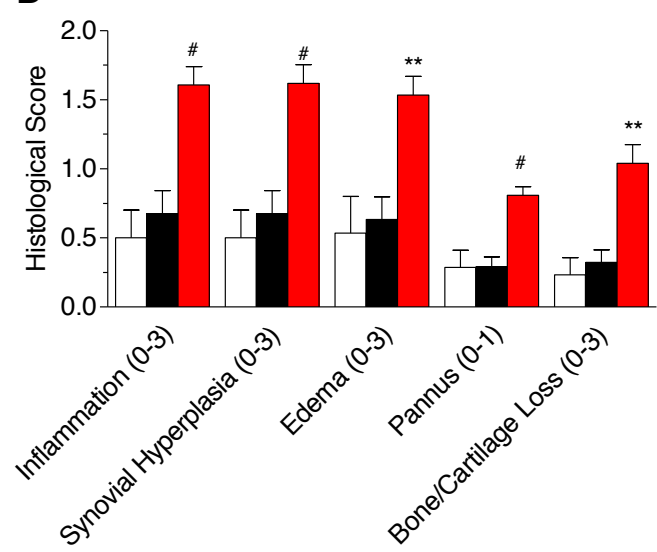

E

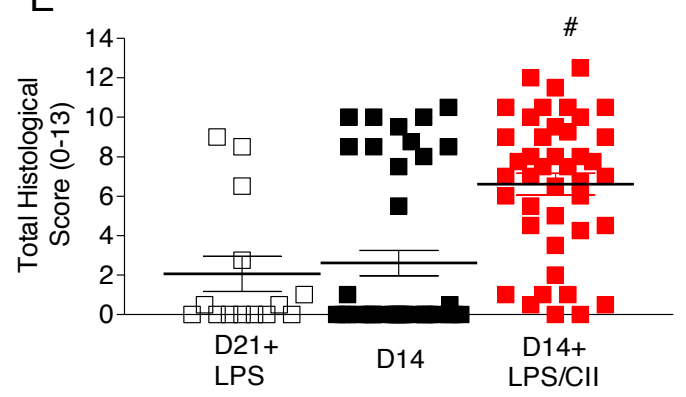

Figure 2. The addition of lipopolysaccharide (LPS) plus collagen type II (CII) markedly increased arthritis severity in an specific-pathogen free (SPF) colony. DBA/1 mice housed in an SPF colony were immunized as per protocols D21+LPS $(n=8)$, D14 $(n=21)$, and D14+LPS/CII $(n=22)$ and then followed for the development of arthritis. Arthritis was defined as the development of joint swelling and was measured as (A) the percentage of mice that have any swollen joints. The average clinical arthritis score (mean \pm SEM) for each group is shown in (B). (C) Final week arthritis scores $>0$ demonstrate the severity of arthritis when it does occur (bar is mean and whiskers are SEM). Histopathology scoring of hind leg knees harvested on day 55 for individual parameters (D) indicated as mean (bars) and SEM (error bar) with total histopathological Scores (E) indicated as individual symbols with the line indicating the mean. ${ }^{*} P$-value $<0.05,{ }^{*} P$-value $<0.005, \# P$-value $<0.0005$ in comparison to D21+LPS.

$10 \mu \mathrm{g} / \mathrm{mL}$ of $\mathrm{Cll}$ in $1 \mathrm{M}$ TRIS, $\mathrm{pH} 7.2$ solution at $50 \mu \mathrm{L}$ per well overnight at $4^{\circ} \mathrm{C}$. After 6 washes with $1 \%$ Tween 20 in 1 M TRIS, pH 7.2 solution, duplicate wells were loaded with 50 $\mu \mathrm{L}$ of serum specimens with 7 serial dilutions. The diluent used was $1 \mathrm{M}$ TRIS, pH 7.2, 1\% Tween 20 with 10\% (v/v) Pierce SuperBlock (Thermo Fisher Scientific, Waltham, MA). After 1 hour at room temperature, plates were washed, and $50 \mu \mathrm{l} /$ well HRP-rat anti-mouse lgG1 (catalog \#559626; BD Biosciences, San Jose, CA), HRP-rat anti-mouse lgG2a/c (catalog \#553391; BD Biosciences), biotinrat anti-mouse lgG2b (catalog \#553393; BD Biosciences), or biotin-rat anti-mouse lgG3 (catalog \#553401; BD Biosciences) diluted in the same TRIS/Tween/SuperBlock solution as above was added. After $30 \mathrm{~min}$, all plates were washed, and $50 \mu \mathrm{l} /$ well streptavidinHRP solution (catalog \#21126; Pierce) diluted 1:20,000 in TRIS/Tween/SuperBlock solution was added only to plates containing biotinlabeled secondary antibodies, followed 30 min later by 6 washes. All plates then had 150 $\mu \mathrm{l}$ of a solution consisting of SuperSignaling ELISA Femto Maximum Sensitivity Substrate
Kit (catalog \#37075; Pierce) enhancer and $\mathrm{H}_{2} \mathrm{O}_{2}$ (also included in the kit) plus $1 \mathrm{M}$ TRIS, $\mathrm{pH} 7.2$, in a volume ratio of 1 enhancer: $1 \mathrm{H}_{2} \mathrm{O}_{2}$ : 15 TRIS added to each well and were read on a Fluoroskan luminometer (Luminoskan, Ascent software; Thermo Electron Corporation, Franklin, MA). Results were graphed with inverse of titer on the x-axis using log scale versus optical density (OD) on the y-axis using linear scale. In the region where the lines were linear on the graph for all samples, the lowest possible OD that intersected all samples was chosen, and the corresponding titer was obtained for each sample from the graph. A pooled serum collection from Cll immunized mice was diluted to a similar degree as the test samples and included on each plate. This pooled serum was used as a standard to assure reproducibility and allow comparison of results from plate to plate.

\section{Cll-specific T-cell analysis}

Spleens were harvested on day 56, and 5 $\times 10^{5} \mathrm{cells} /$ well were plated in triplicate at $37^{\circ} \mathrm{C}$ in $96-w e l l$ flat-bottom microtiter plates pre-coated with heat-denatured $\left(56^{\circ} \mathrm{C}\right.$ for
10 min) Cll or antibody 2C11 (anti-CD3)containing cell culture supernatant (from cultured 2C11 hybridoma cells; ATCC, Manassas, VA) as a positive control. Cells were maintained in RPMI (Gibco/Thermo Fisher Scientific) containing 0.5\% normal DBA/1 mouse serum. Supernatant was harvested after $24 \mathrm{~h}$ and interferon (IFN)- $\gamma$ was measured by a standard ELISA technique as previously described (mouse IFN- $\gamma$ OptEIA kit, catalogue \#555138; BD Biosciences, San Jose, CA) (28).

\section{Statistics}

The Mann-Whitney $t$-test was used to compare non-parametric data from clinical arthritis scores, histopathologic scores, and immunoglobulin titers using GraphPad Prism (GraphPad Software, La Jolla, CA).

\section{Results and discussion}

Development of CIA in an SPF compared with a conventional facility: Decreased arthritis susceptibility in mice housed in SPF facilities as compared with conventional facilities has been observed by ourselves (Figure 1) and 
others (20). A typical CIA protocol referred to as D21 in Table 1 results in 80\%-100\% of mice exhibiting severe arthritis in conventional facilities (29). In our SPF facility, this typical protocol results in only $10 \%$ of mice exhibiting arthritis (Figure 1).

Adding LPS to increase the development of CIA in an SPF facility: To increase the incidence of arthritis, many investigators have used LPS to stimulate innate immunity and invoke a more vigorous inflammatory reaction $(7,16,24,25,30,31)$. To determine whether addition of LPS to the D21 protocol in an SPF facility would increase the incidence of arthritis, LPS was administered 3 days after the second dose of CII/CFA (D21+LPS, Table 1). LPS significantly increased the incidence and severity of arthritis (Figure 2) in mice housed in the SPF facility, but still to a lesser degree than in the conventional facility (Figure 1).

Elimination of 1 week between CII/CFA immunizations: To investigate the effect of shortening the interval between CII/CFA immunizations from 3 weeks to 2 weeks on the development of arthritis, the D21+LPS protocol was compared with a protocol with Cll/CFA being given at days 0 and 14 and LPS given 3 days following the second immunization (D14 protocol, Table 1). The D21+LPS and D14 protocols produced a similar incidence and severity of arthritis (Figure 2), illustrating that elimination of 1 week between Cll/CFA injections does not diminish development of arthritis.

Addition of a $3^{\text {rd }}$ and $4^{\text {th }}$ immunization of Cll in conjunction with LPS: We hypothesized that additional CII and LPS (LPS/CII) doses following the two initial Cl//CFA doses would increase the Cll-specific immune response and result in more severe arthritis. The D14 protocol with inclusion of two LPS/Cll injections (D14+LPS/Cll protocol, Table 1) had an increased incidence of arthritis (Figure 2A), a higher maximum mean arthritis score (Figure 2B), more severe arthritis when arthritis did occur (Figure 2C), and increases in all histopathological scores (Figure 2, D and E) compared with D14.

IFA substituted for CFA: To limit CFA use, we investigated whether IFA could be substituted for CFA in either the second immunization (CFA/IFA+LPS/Cll protocol, Table 1) or both immunizations (IFA/IFA+LPS/CII protocol, Table 1) and still invoke significant IA. The highest incidence and most severe arthritis were observed in mice receiving CFA in at least one of the first two Cll injections (Figure 3, A-E). Nevertheless, even mice receiving IFA in both of the first 2 injections

\begin{tabular}{|lll|}
\hline$\square$ D14 & D14+LPS/CII & CFA/IFA+LPS/CII \\
$\square$ IFA/IFA+LPS/CII & IFA/IFA+CII & $\square$ IFA/IFA+LPS \\
\hline
\end{tabular}

A

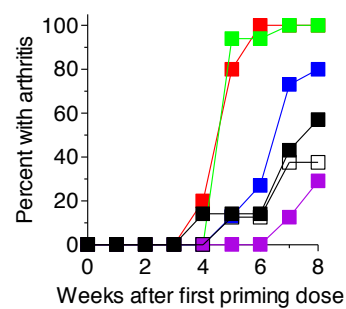

B
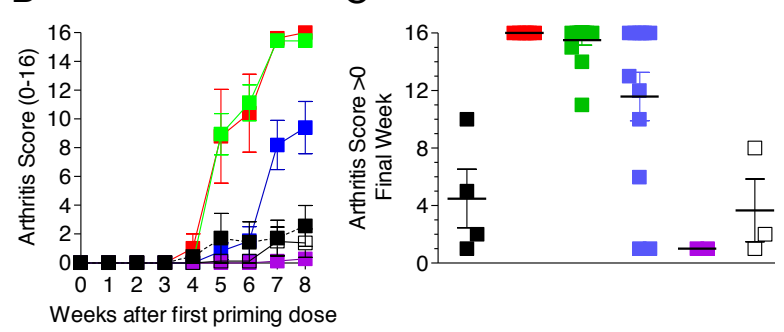

D

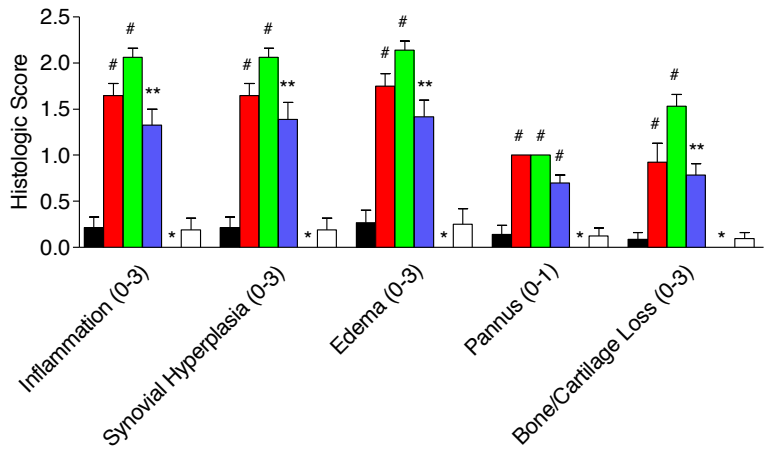

E

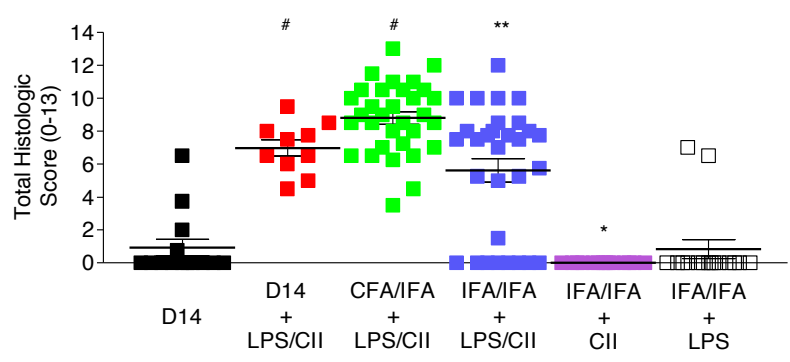

F

Day 2
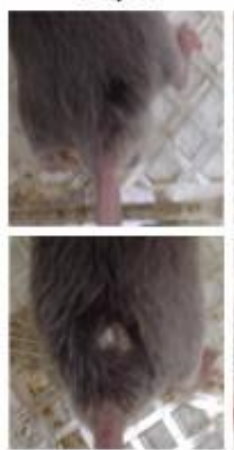

Day 8
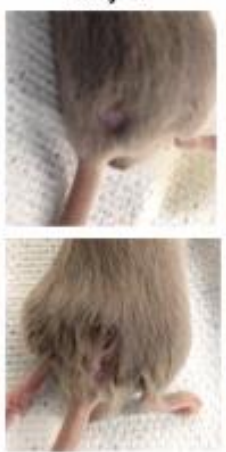

Day 15

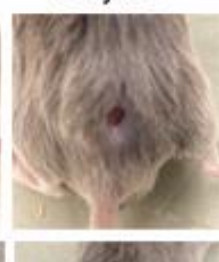

Day 21

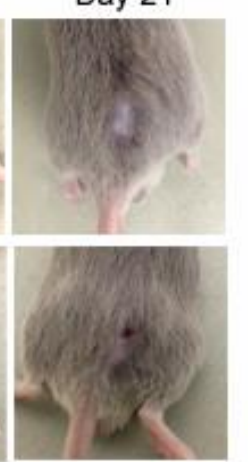

Figure 3. Immunization of mice with additional doses of lipopolysaccharide (LPS) + collagen type II (CII) eliminates the need for complete Freund's adjuvant (CFA) to invoke substantial arthritis. (A-E) Specific-pathogen free (SPF) colony-housed DBA/1 mice were injected per various protocols (see Table 1 for more details), and arthritis was measured as in Figure 2. $n=$ 6 -16/group. * $P$-value $<0.05$, ${ }^{*} P$-value $<0.005$, and $\# P$-value $<0.0005$ in comparison to D14. (F) Pictures of typical skin lesions in different mice at the site of CII/CFA injection over time.

followed by 2 doses of LPS/Cll had an $80 \%$ incidence of arthritis and exhibited very severe arthritic disease with a mean total histopathology score of 5.6 compared with 7.0 and 8.8 for the D14+LPS/CII and CFA/IFA+LPS/ Cll protocol groups, respectively (Figure 3E).
Immunization of LPS along with Cll: Since LPS/Cll given at later stages of the disease increased arthritic incidence and severity, it was of interest to determine whether immunization with either one alone on days 28 and 42 would be sufficient for development of severe 
A

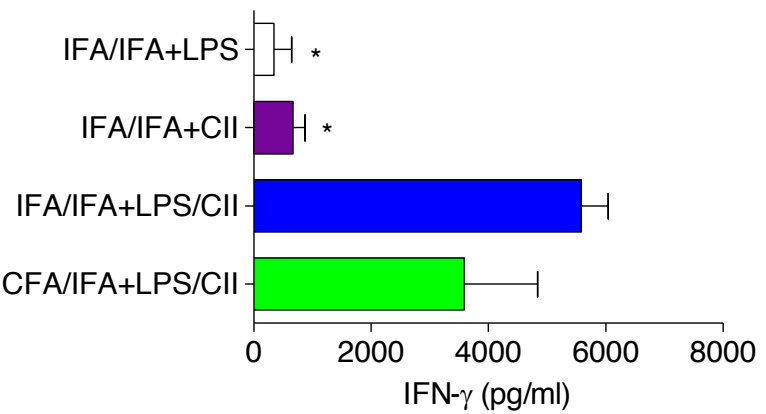

B

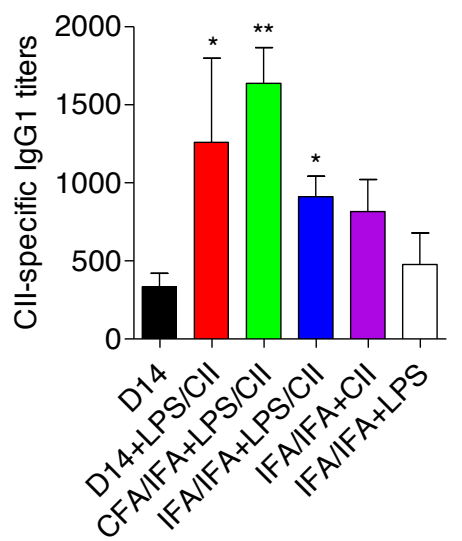

D

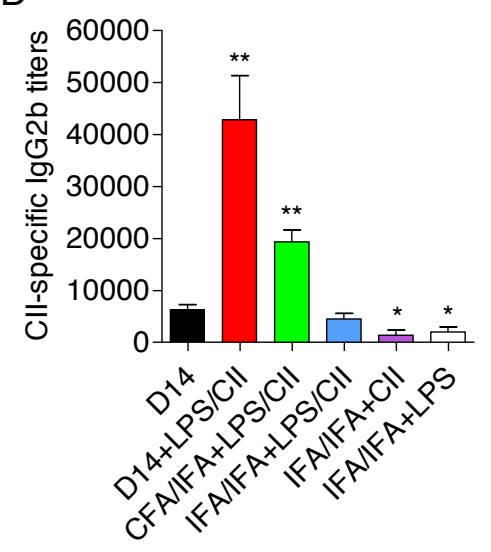

C

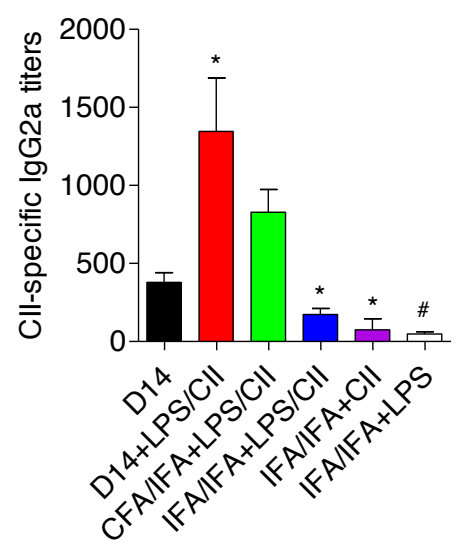

$\mathrm{E}$

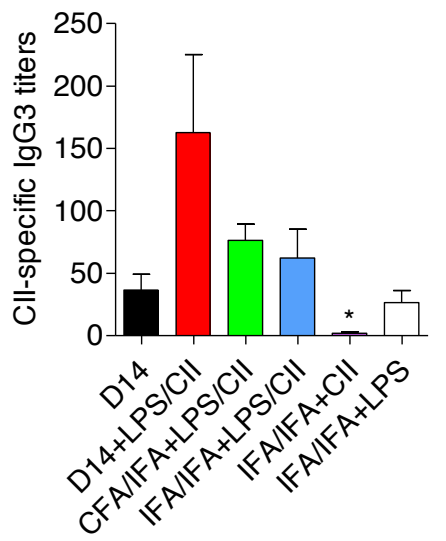

Figure 4. Spleens produce larger amounts of interferon (IFN)- $\gamma$ and collagen type II (CII)-specific IgG isotype titers increase with the addition of lipopolysaccharide (LPS)/CII to the induction protocol. (A) Spleen cells were harvested on day 56 from 4 mice from the indicated protocol groups in Figure 3, cultured for $24 \mathrm{~h}$, and stimulated with CII. Culture media was collected and IFN- $\gamma$ concentration was measured by ELISA. $n=4$ /group. ${ }^{*} P$-value $<0.05$ in comparison to protocol CFA/ IFA+LPS/CII. Serum was collected from mice from labeled protocol groups in Figure 3 on day 35 and measured by ELISA for Cll-specific IgG1 (B), IgG2a/c (C), IgG2bY (D), and IgG3 (E). $n=5-16 /$ group. ${ }^{*} P$-value $<0.05,{ }^{*} *$-value $<0.005$, and $\# P$-value $<0.0005$ in comparison to protocol D14.

arthritis. However, neither alone offered any additional degree of arthritis induction over the D14 protocol for which neither additional LPS nor Cll is given (Figure 3, A-E).

Enhanced Cll-specific B- and T-cell activation following LPS and $C / l$ treatment with the IFA/IFA protocol: To determine whether the enhanced macroscopic and histological CIA observed with additional CI/LPS treat- ments resulted in a concordant increase in Cll-specific adaptive immune responses, assays of T-cell (spleen cultures) and B-cell function (Cll-specific immunoglobulin titers) were performed. Stimulation of the cultured spleen cells by $\mathrm{Cll}$ demonstrated increased IFN- $\gamma$ production by the CFA/IFA+LPS/CII and IFA/IFA+LPS/CII groups compared with either the IFA/IFA+LPS or IFA/IFA+CII groups, suggesting a more vigorous T-cell response in mice receiving both LPS and Cll together (Figure 4A) and correlating well with both the arthritic and histopathological scores in Figure 3. The Cll-specific B-cell response, as measured by serum levels of anti-Cll antibody titers for IgG1, 2a, 2b, and 3, also demonstrated a similar marked increase in activation to the combination of LPS and Cll as compared with individual LPS or Cll (Figure 4, B-E) in arthritis and histopathological scores in Figure 3, B-E.

Numerous variations to the murine CIA model protocol have been published, but no formal report has compared the methods head-to-head or assessed how minor variations in these protocols could be used to invoke changes in the severity and incidence of arthritis. The development of simple variations in the original protocol that allow investigators to induce various grades of arthritis severity in a murine model of IA is extremely useful, depending on the goal of the study; more severe arthritis could be used when assessing the ability of an inhibitor of arthritis, whereas a mild arthritis model could be more useful in assessing an exacerbating factor. A typical CIA protocol, D21, where Cll in CFA is given i.d. in 2 separate injections 21 days apart is very similar to the majority of published protocols for CIA (17-20). However, this "standard protocol" results in mild arthritis in our SPF colonies even in highly susceptible DBA/1 mice (Figure 1). Addition of LPS alone 3 days after the second dose of CII/CFA modestly increased the incidence of arthritis in SPF-housed mice (Figure 2) but it was still much less compared with conventionally housed mice (Figure 1). Addition of LPS plus Cll 14 and 28 days after the second dose of Cll/CFA resulted in 100\% of SPF-housed mice exhibiting severe disease similar to published studies using the standard protocol, D21, with DBA/1 mice in a conventional colony (Figures 1 and 2). Shortening of the interval between doses of CII/CFA from 21 days to 14 days did not change our results and offers the advantage of a shorter protocol time for the D14+LPS/CII protocol (Figure 2). Of note, these findings may not translate directly to other SPF colonies, as various SPF facilities may not be equivalent $(32,33)$; however, the results from this protocol can serve as a guide for such studies.

A protocol that invokes severe arthritis in nearly $100 \%$ of the mice will be very useful for evaluating the role of crucial receptors and molecules in the development and pathogenesis of CIA, as well as potential thera- 
peutics, but may be too severe to appreciate more subtle involvement or partial, yet important, inhibition by certain treatments. It also has the disadvantage of using CFA, an adjuvant that causes local ulcerations at injection sites and is under significant scrutiny by regulatory agencies that aim to limit its use (http://grants.nih.gov/grants/ olaw/olaw.htm). Our own experience is that some mice given CII/CFA, with or without LPS given later, develop lesions at the CFA injection site within a few days and then scar over in a few weeks (Figure 3F). Consequently, an exploration of discrete variations of this protocol was undertaken to uncover more graded responses along with the realization that IFA as a substitute for CFA often avoids this reaction but with the trade-off of not evoking as significant of an immune response. Despite this less vigorous reaction, substituting IFA in place of CFA allowed for development of arthritis with disease severity and incidence intermediate between the D14 and D14+LPS/CII protocols and allows for substitution of IFA for CFA (Figure 3B).

Elimination of either LPS or Cll from the subsequent day 28 and 42 injections resulted in similar IA to the D14 protocol (Figure 3,
A-E) thus offering no advantage over total elimination of both. Presumably LPS and CII function in concert to further drive the immune reaction and subsequent increase in arthritic development. LPS is expected to serve as a non-specific stimulant of the immune system by promoting release of various inflammatory cytokines and stimulating antibody secretion (7). Despite this non-specific inflammation, repeated dosing of LPS alone beyond the day 17 dose does not promote any additional activation of the $\mathrm{T}$ - or B-cell pathology directed against joint collagen, as demonstrated in Figure 4. A paradoxical finding was that $\mathrm{Cll}$ alone did not induce additional T- or B-cell activation (Figure 4) but instead decreased the severity of arthritis. One possible explanation for this result may be that non-joint-associated injected Cll may act as a scavenger for available Cll-specific antibodies, thus preventing their binding with joint-associated Cll and preventing concomitant local T-cell activation, joint inflammation, and pathology. This scavenger explanation could be likened to part of the theoretic basis of "rush desensitization" used in rapid allergy treatment where a cumulative large dose of Ag given in multiple doses over a relatively short period of time quickly occupies most

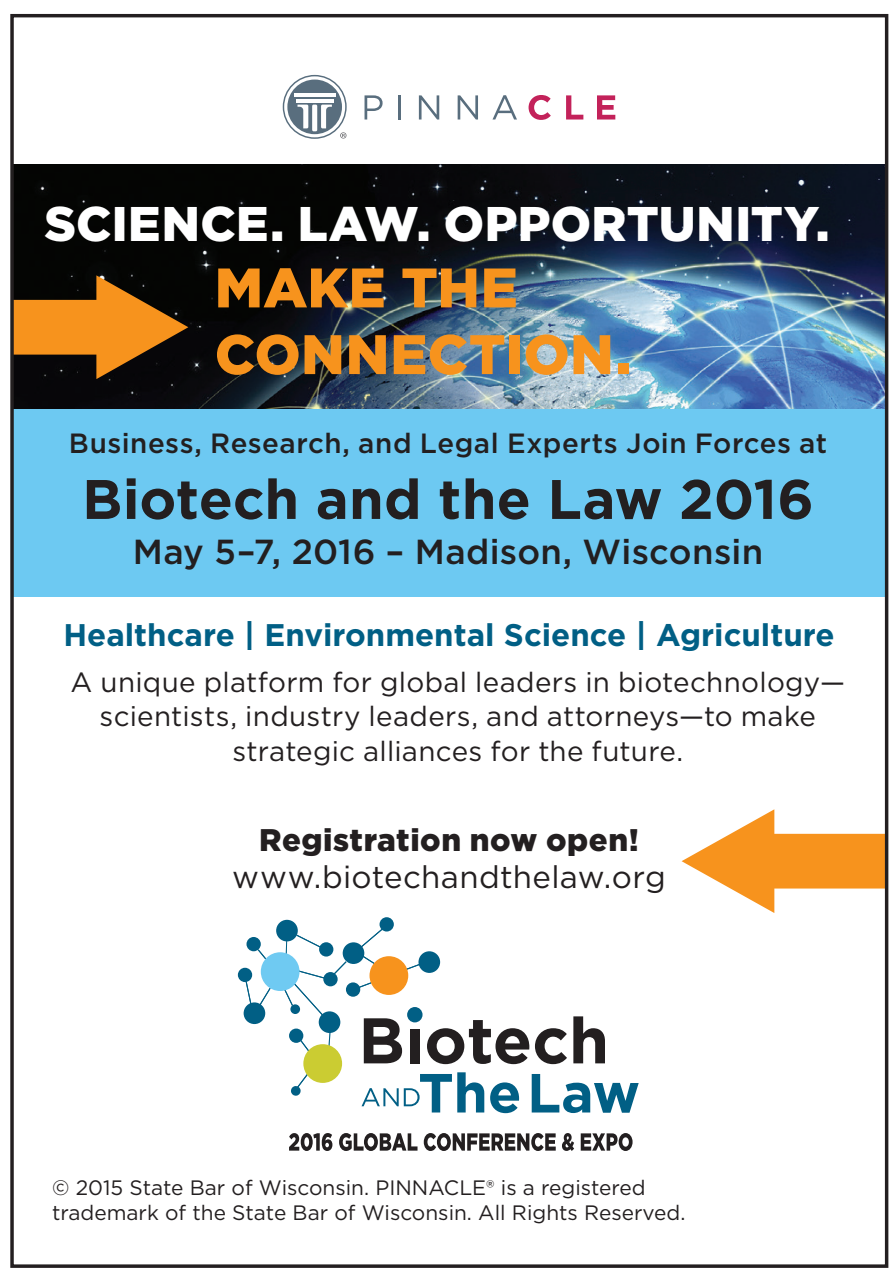

available Ag-specific antibody binding sites, thus allowing for further Ag exposure without induction of life-threatening allergic symptoms $(34,35)$. Regardless, Cll when given with LPS appears to guide the non-specific inflammatory response raised by LPS in a directed fashion toward Cll-specific T-cell release of additional IFN- $\gamma$ and B-cell secretion of additional $\mathrm{Cll}$-specific lgG1, IgG2a, and lgG2b (Figure 4) and more severe pathology (Figure 3, D and E).

The severity of arthritis for each variation of the protocol was closely correlated with the overall B- and T-cell responses as measured by Cll-specific ELISA and in vitro cultured spleen cell production of IFN- $\gamma$ in response to Cll stimulation (Figure 4). This result is consistent with many reports alluding to the importance of both B- and T-cell activation on CIA development (36-42) and offers an additional measure of arthritic response to the various protocols.

Another important factor in induction of arthritis is the animal gut flora, which has been shown to influence the incidence and severity of IA $(43,44)$. Notably, variation in gut flora exists from SPF facility to SPF facility and among various strains of mice $(32,45$ 48). Therefore, similar studies performed in other facilities may not provide results exactly as presented here, but general findings should be similar.

Our findings provide a panel of protocols for investigators to use in designing experiments to maximize the utility of the results depending on whether exacerbating or enhancing aspects of arthritis is to be expected, thus limiting the number of animals needed. These protocols demonstrate a reasonable role for the substitution of IFA for CFA under many circumstances. With the development of siRNA and CRISPR technology, the potential of these protocols in $\mathrm{DBA} / 1$ background mice is tremendous.

\section{Author contributions}

Both authors were involved with all aspects of the study.

\section{Acknowledgments}

We would like to thank Ashley Hall for taking care of the experimental animals and her assistance with experiments, and William Ridgway and Matthew Flick for their helpful review of the manuscript. This work was supported in part by the Cincinnati Rheumatic Disease Core Center, Animal Models of Inflammatory Disease Core, $\mathrm{NIH}$ 
AR47363 (P30 AR47363 to RTS). This paper is subject to the NIH Public Access Policy.

\section{Competing interests}

The authors declare no competing interests.

\section{References}

1. Holmdahl, R., R. Bockermann, J. Backlund, and H. Yamada. 2002. The molecular pathogenesis of collagen-induced arthritis in mice--a model for rheumatoid arthritis. Ageing Res. Rev. 1:135-147.

2. Kannan, K., R.A. Ortmann, and D. Kimpel. 2005. Animal models of rheumatoid arthritis and their relevance to human disease. Pathophysiology 12:167-181.

3. Cho, Y.G., M.L. Cho, S.Y. Min, and H.Y. Kim. 2007. Type II collagen autoimmunity in a mouse model of human rheumatoid arthritis. Autoimmun. Rev. 7:65-70.

4. Billiau, A. and P. Matthys. 2011. Collageninduced arthritis and related animal models: how much of their pathogenesis is auto-immune, how much is auto-inflammatory? Cytokine Growth Factor Rev. 22:339-344.

5. Holmdahl, R., M.E. Andersson, T.J. Goldschmidt, L. Jansson, M. Karlsson, V. Malmstrom, and J. Mo. 1989. Collagen induced arthritis as an experimental model for rheumatoid arthritis. Immunogenetics, pathogenesis and autoimmunity. APMIS. 97:575-584.

6. Myers, L.K., E.F. Rosloniec, M.A. Cremer, and A.H. Kang. 1997. Collagen-induced arthritis, an animal model of autoimmunity. Life Sci. 61:18611878.

7. Tanaka, S., T. Toki, T. Akimoto, and K. Morishita. 2013. Lipopolysaccharide accelerates collagen-induced arthritis in association with rapid and continuous production of inflammatory mediators and anti-type II collagen antibody. Microbiol. Immunol. 57:445-454.

8. Wooley, P.H., J. Dutcher, M.B. Widmer, and S. Gillis. 1993. Influence of a recombinant human soluble tumor necrosis factor receptor $\mathrm{FC}$ fusion protein on type II collagen-induced arthritis in mice. J. Immunol. 151:6602-6607.

9. Williams, R.O., J. Ghrayeb, M. Feldmann, and R.N. Maini. 1995. Successful therapy of collagen-induced arthritis with TNF receptor-IgG fusion protein and combination with anti-CD4. Immunology 84:433-439.

10. Williams, R.O., M. Feldmann, and R.N. Maini. 1992. Anti-tumor necrosis factor ameliorates joint disease in murine collagen-induced arthritis. Proc. Natl. Acad. Sci. USA 89:9784-9788.

11. Wang, Q.T., Y.J. Wu, B. Huang, Y.K. Ma, S.S. Song, L.L. Zhang, J.Y. Chen, H.X. Wu, et al. 2013. Etanercept attenuates collagen-induced arthritis by modulating the association between BAFFR expression and the production of splenic memory B cells. Pharmacol Res. 68:38-45.

12. Yang, S., A.M. Hollister, E.A. Orchard, S.I. Chaudhery, D.V. Ostanin, S.J. Lokitz, and J.M. Mathis. 2013. Quantification of bone changes in a collagen-induced arthritis mouse model by reconstructed three dimensional micro-CT. Biol. Proced. Online 15:8.

13. Nozawa, K., M. Fujishiro, M. Kawasaki, A. Yamaguchi, K. Ikeda, S. Morimoto, K. Iwabuchi, M. Yanagida, et al. 2013. Inhibition of connective tissue growth factor ameliorates disease in a murine model of rheumatoid arthritis. Arthritis Rheum. 65:1477-1486.

14. Evans, E.K., R. Tester, S. Aslanian, R. Karp, M. Sheets, M.T. Labenski, S.R. Witowski, H. Lounsbury, et al. 2013. Inhibition of Btk with CC-292 provides early pharmacodynamic assessment of activity in mice and humans. J. Pharmacol. Exp. Ther. 346:219-228.

15. Dowty, M.E., M.I. Jesson, S. Ghosh, J. Lee, D.M. Meyer, S. Krishnaswami, and N. Kishore. 2014. Preclinical to clinical translation of tofacitinib, a Janus kinase inhibitor, in rheumatoid arthritis. J. Pharmacol. Exp. Ther. 348:165-173.

16. Caccese, R.G., J.L. Zimmerman, and R.P. Carlson. 1992. Bacterial lipopolysaccharide potentiates type II collagen-induced arthritis in mice. Mediators Inflamm. 1:273-279.

17. Brand, D.D., K.A. Latham, and E.F. Rosloniec. 2007. Collagen-induced arthritis. Nat. Protoc. 2:12691275.

18. Williams, R.O. 2004. Collagen-induced arthritis as a model for rheumatoid arthritis. Methods Mol. Med. 98:207-216.

19. Brand, D.D., A.H. Kang, and E.F. Rosloniec. 2004. The mouse model of collagen-induced arthritis. Methods Mol. Med. 102:295-312.

20. Bevaart, L., M.J. Vervoordeldonk, and P.P. Tak. 2010. Collagen-induced arthritis in mice. Methods Mol. Biol. 602:181-192.

21. Terato, K., D.S. Harper, M.M. Griffiths, D.L. Hasty, X.J. Ye, M.A. Cremer, and J.M. Seyer. 1995. Collagen-induced arthritis in mice: synergistic effect of E. coli lipopolysaccharide bypasses epitope specificity in the induction of arthritis with monoclonal antibodies to type II collagen. Autoimmunity 22:137-147.
22. Reiff, A., M. Zastrow, B.C. Sun, S. Takei, H. Mitsuhada, B. Bernstein, and D.L. Durden. 2001. Treatment of collagen induced arthritis in DBA/1 mice with L-asparaginase. Clin. Exp. Rheumatol. 19:639646 .

23. Yoshino, S. and M. Ohsawa. 2000. The role of lipopolysaccharide injected systemically in the reactivation of collagen-induced arthritis in mice. $\mathrm{Br}$. J. Pharmacol. 129:1309-1314.

24. Yoshino, S., E. Sasatomi, Y. Mori, and M. Sagai. 1999. Oral administration of lipopolysaccharide exacerbates collagen-induced arthritis in mice. J. Immunol. 163:3417-3422

25. Joosten, L.A., E. Lubberts, P. Durez, M.M. Helsen, M.J. Jacobs, M. Goldman, and W.B. van den Berg. 1997. Role of interleukin-4 and interleukin-10 in murine collagen-induced arthritis. Protective effect of interleukin-4 and interleukin-10 treatment on cartilage destruction. Arthritis Rheum. 40:249-260.

26. Qi, X., M.J. Flick, M. Frederick, Z. Chu, R. Mason, M. DeLay, and S. Thornton. 2012. Saposin C coupled lipid nanovesicles specifically target arthritic mouse joints for optical imaging of disease severity. PLoS ONE 7:e33966.

27. Raghu, H., A. Jone, C. Cruz, C.L. Rewerts, M.D. Frederick, S. Thornton, J.L. Degen, and M.J. Flick. 2014. Plasminogen is a joint-specific positive or negative determinant of arthritis pathogenesis in mice. Arthritis Rheumatol. 66:1504-1516.

28. Thornton, S., G.P. Boivin, K.N. Kim, F.D. Finkelman, and R. Hirsch. 2000. Heterogeneous effects of IL-2 on collagen-induced arthritis. J. Immunol. 165:1557-1563.

29. Flick, M.J., A.K. Chauhan, M. Frederick, K.E. Talmage, K.W. Kombrinck, W. Miller, E.S. Mullins, J.S. Palumbo, et al. 2011. The development of inflam-

\section{P $\Lambda^{\mathrm{m}} \begin{aligned} & \text { Integrated Patch Clamp Amplifier } \\ & \text { And Data Acquisition System }\end{aligned}$}

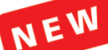

The IPA ${ }^{\text {TM }}$ combines a low-noise patch clamp amplifier with onboard D/A conversion and data acquisition via high-speed USB into a single integrated system with SutterPatch ${ }^{\text {TM }}$, a comprehensive software package. Backed by the support and service you've come to expect from Sutter Instrument at an introductory price.

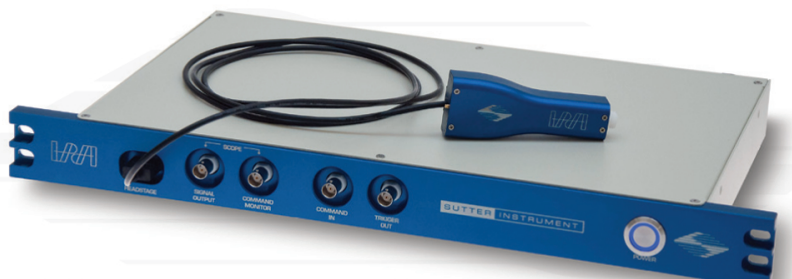

- Fully integrated - ensuring quick and easy setup.

- Optimized for whole-cell patch clamp recordings in tissue slices, adherent or dissociated cells.

- Full computer control provides automated compensation of electrode and whole-cell capacitance.

- Voltage and true current clamp capability for complete characterization of cells' electrical activity.

- SutterPatch ${ }^{\mathrm{Tm}}$ software excels in comprehensive data management, intuitive navigation and streamlined data analysis.

\begin{tabular}{l|l}
\hline$S U T E R$ & INSTRUMENT
\end{tabular} 
matory joint disease is attenuated in mice expressing the anticoagulant prothrombin mutant W215A/E217A. Blood 117:6326-6337.

30. Gould, D.J., C. Bright, and Y. Chernajovsky. 2004. Inhibition of established collagen-induced arthritis with a tumour necrosis factor-alpha inhibitor expressed from a self-contained doxycycline regulated plasmid. Arthritis Res. Ther. 6:R103-R113.

31. Kang, I., W.W. Lee, and Y. Lee. 2000. Modulation of collagen-induced arthritis by $\mathrm{IL}-4$ and dexamethasone: the synergistic effect of IL-4 and dexamethasone on the resolution of CIA. Immunopharmacology 49:317-324.

32. Hufeldt, M.R., D.S. Nielsen, F.K. Vogensen, T. Midtvedt, and A.K. Hansen. 2010. Variation in the gut microbiota of laboratory mice is related to both genetic and environmental factors. Comp. Med. 60:336-347.

33. Besselsen, D.G., C.L. Franklin, R.S. Livingston, and L.K. Riley. 2008. Lurking in the shadows: emerging rodent infectious diseases. ILAR J. 49:277290.

34. Cernadas, J.R. 2013. Desensitization to antibiotics in children. Pediatr Allergy Immunol. 24:3-9.

35. Stämpfli, M.R., M. Rudolf, S. Miescher, J.M. Pachlopnik, and B.M. Stadler. 1995. Antigenspecific inhibition of lgE binding to the high-affinity receptor. J. Immunol. 155:2948-2954.

36. Alzabin, S. and R.O. Williams. 2011. Effector T cells in rheumatoid arthritis: lessons from animal models. FEBS Lett. 585:3649-3659.

37. Dunussi-Joannopoulos, K., G.E. Hancock, A. Kunz, M. Hegen, X.X. Zhou, B.J. Sheppard, J. Lamothe, E. Li, et al. 2005. B-cell depletion inhibits arthritis in a collagen-induced arthritis (CIA) model, but does not adversely affect humoral responses in a respiratory syncytial virus (RSV) vaccination model. Blood 106:2235-2243.

38. Wang, H., S.A. Marsters, T. Baker, B. Chan, W.P. Lee, L. Fu, D. Tumas, M. Yan, et al. 2001. TAClligand interactions are required for $\mathrm{T}$ cell activation and collagen-induced arthritis in mice. Nat. Immunol. 2:632-637.

39. Corthay, A., A. Johansson, M. Vestberg, and R. Holmdahl. 1999. Collagen-induced arthritis development requires alpha beta T cells but not gamma delta T cells: studies with T cell-deficient (TCR mutant) mice. Int. Immunol. 11:1065-1073.

40. Seetharaman, R., A.L. Mora, G. Nabozny, M. Boothby, and J. Chen. 1999. Essential role of T cell NF-kappa B activation in collagen-induced arthritis. J. Immunol. 163:1577-1583.

41. Yanaba, K., Y. Hamaguchi, G.M. Venturi, D.A. Steeber, E.W. St Clair, and T.F. Tedder. 2007. B cell depletion delays collagen-induced arthritis in mice: arthritis induction requires synergy between humoral and cell-mediated immunity. J. Immunol. 179:1369-1380.

42. Inglis, J.J., G. Criado, M. Medghalchi, M. Andrews, A. Sandison, M. Feldmann, and R.O. Williams. 2007. Collagen-induced arthritis in C57BL/6 mice is associated with a robust and sustained T-cell response to type II collagen. Arthritis Res. Ther. 9:R113.

43. Dorozynska, I., M. Majewska-Szczepanik, K. Marcinska, and M. Szczepanik. 2014. Partial depletion of natural gut flora by antibiotic aggravates collagen induced arthritis (CIA) in mice. Pharmacol Rep. 66:250-255.

44. Wu, H.J., I.I. Ivanov, J. Darce, K. Hattori, T. Shima, Y. Umesaki, D.R. Littman, C. Benoist, and D. Mathis. 2010. Gut-residing segmented filamentous bacteria drive autoimmune arthritis via T helper 17 cells. Immunity 32:815-827.

45. Vaahtovuo, J., P. Toivanen, and E. Eerola. 2003. Bacterial composition of murine fecal microflora is indigenous and genetically guided. FEMS Microbiol. Ecol. 44:131-136.

46. Buhnik-Rosenblau, K., Y. Danin-Poleg, and Y. Kashi. 2011. Predominant effect of host genetics on levels of Lactobacillus johnsonii bacteria in the mouse gut. Appl. Environ. Microbiol. 77:6531-6538.

47. Taneja, V. 2014. Arthritis susceptibility and the gut microbiome. FEBS Lett. 588:4244-4249.

48. Ivanov, I.I., K. Atarashi, N. Manel, E.L. Brodie, T. Shima, U. Karaoz, D. Wei, K.C. Goldfarb, et al. 2009. Induction of intestinal Th17 cells by segmented filamentous bacteria. Cell 139:485-498.

Received 07 October 2015; accepted 16 December 2015.

Address correspondence to Richard T. Strait, Department of Pediatrics and Cincinnati Children's Hospital Medical Center, Division of Emergency Medicine, University of Cincinnati College of Medicine, 3333 Burnet Avenue, Cincinnati, OH 45229. E-mail: rick.strait@cchmc.org

To purchase reprints of this article, contact: biotechniques@fosterprinting.com

\section{MYCOPLASMA CONTROL REAGENTS}

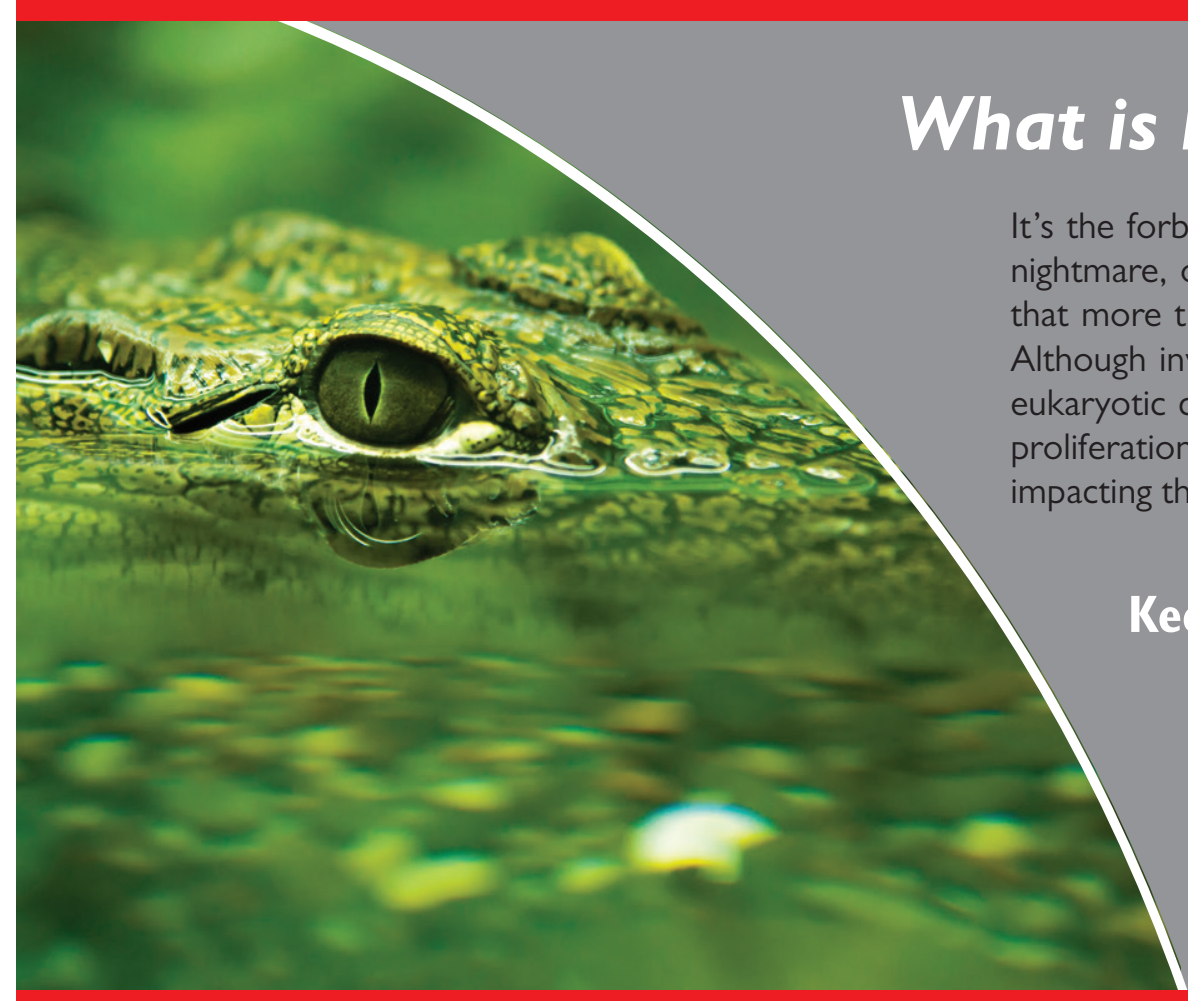

Keep your cells mycoplasma-free:

Detect: PlasmoTest ${ }^{\mathrm{TM}}$

Eradicate: Plasmocin ${ }^{\mathrm{TM}}$ - Plasmocure ${ }^{\mathrm{TM}}$

Prevent: Normocin ${ }^{\mathrm{TM}}$ - Normocure ${ }^{\mathrm{TM}}$ 